\title{
Alzheimer's theory makes a splash
}

\section{Neuroscientists probe idea that neuronal pruning may contribute to degenerative disorder.}

The Alzheimer's research community is buzzing about a theory suggesting that a close relative of the $\beta$-amyloid protein, and not necessarily $\beta$-amyloid itself - the long-standing suspect - may be a major culprit in the disease.

The theory holds that an amyloid-related mechanism that prunes neuronal connections in the brain in the fast-growth phase of early life may be triggered by ageing-related processes in later life to cause the neuronal withering of Alzheimer's disease (A. Nikolaev et al. Nature 457, 981-989; 2009).

"We have yet to get a disease-modifying drug that works. So we're missing something, and maybe this is one of the missing pieces," says Donna Wilcock, a neurologist at Duke University in Durham, North Carolina.

"I think people are bored of the amyloid hypothesis and would just love to have something else to follow up," adds John Hardy, a neurologist at University College London.

Insoluble clumps of the $\beta$-amyloid peptide appear in the brains of patients with Alzheimer's, and mutations in amyloid's precursor protein (APP) have been linked to rare, familial forms of the disease. But how amyloid contributes to the damage of Alzheimer's is not clear, and several anti-amyloid drugs have failed in phase III clinical trials (see Nature 456, 161-

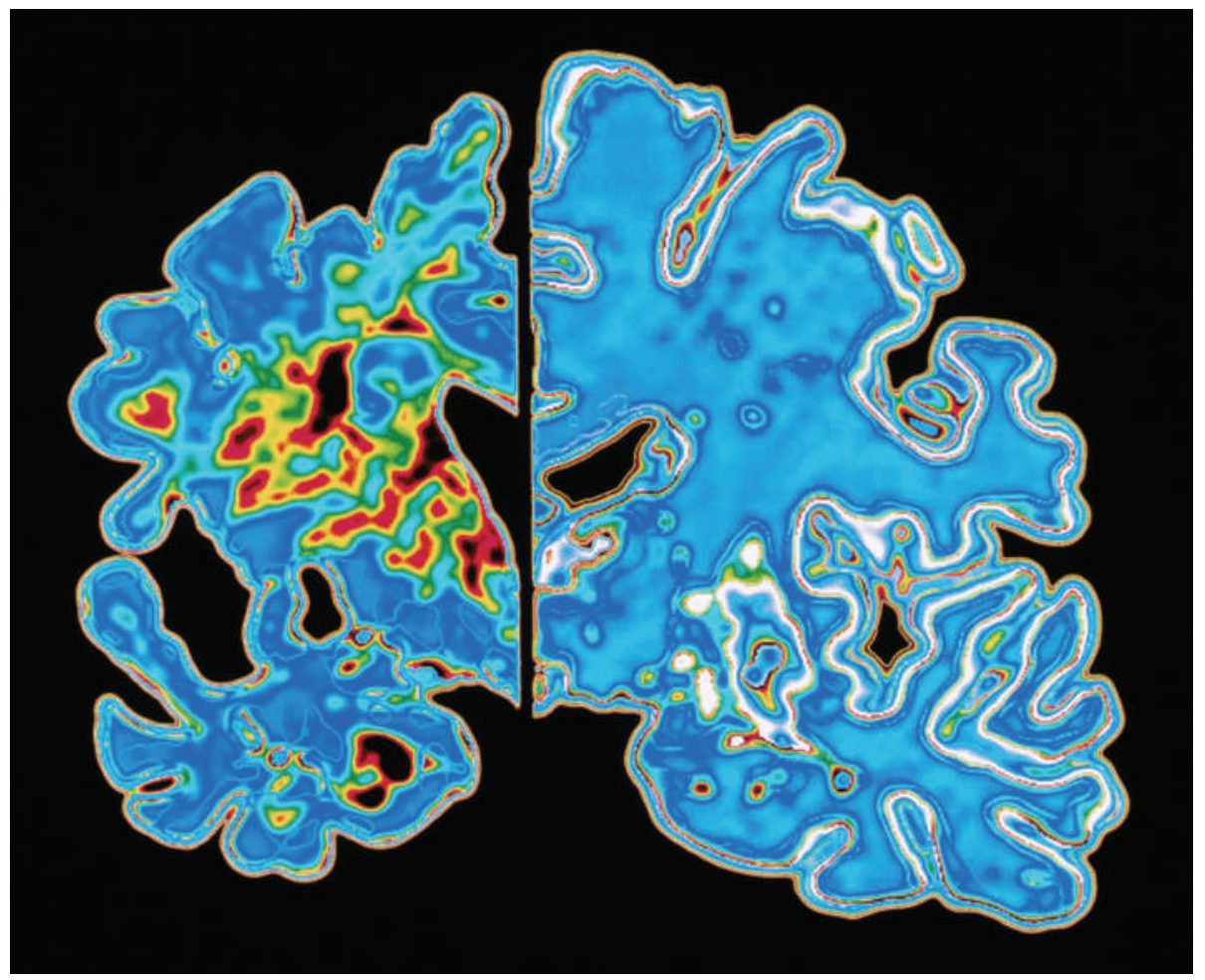

The brain of a patient with Alzheimer's disease (left) is shrunken compared with a normal brain (right). $164 ; 2008)$. Some scientists are unsure that any form of $\beta$-amyloid contributes to much of the neuronal destruction.

Now, researchers suggest that Alzheimer's might be caused by an APP-derived protein - just not necessarily $\beta$-amyloid. The paper describes a naturally occurring brain-pruning process and identifies one of its key players: $\mathrm{N}$-APP, a fragment of APP that is adjacent to $\beta$-amyloid and is cleaved from APP by one of the same enzymes.

N-APP triggers the self-destruct pathway by binding to a neuronal receptor called DR6 (death receptor 6). DR6 is highly expressed in the human brain regions most affected by Alzheimer's, so it is possible that the N-APP/ DR6 pathway might be hijacked in the ageing brain to cause damage.

The lead author of the study, Marc Tessier-Lavigne of Genentech, a biotechnology company in
"It opens up a lot of lines of new research for us." play a part too, perhaps by depressing synaptic function, as other research suggests. "The two are not mutually exclusive," he says.

In any case, N-APP's relevance to Alzheimer's is not yet proved. Bruce Yankner, a neurologist at Harvard University, says that the study focused on embryonic cell types, which are not affected in Alzheimer's disease. (Tessier-Lavigne says that his lab hopes to publish data soon on N-APP/ DR6 mechanisms in Alzheimer's-relevant adult brain cells.)

\section{New model army needed}

There is also no evidence, so far, that the $\mathrm{N}$-APP pruning pathway is active in animal models of Alzheimer's disease. The most commonly used mutant 'Alzheimer's mice' overexpress APP and mimic the amyloid deposition seen in Alzheimer's brains, and therefore presumably overexpress N-APP, too. Yet these mice strikingly don't South San Francisco, California plays down the conflict with the amyloid hypothesis. He emphasizes that even if N-APP does contribute to the withering of neuronal connections in Alzheimer's, $\beta$-amyloid might
The usefulness of these mice as models for human disease has long been questioned, points out Tessier-Lavigne. In initial studies, show Alzheimer's-like neuronal destruction.
N-APP triggered self-destruction only when neurons were also deprived of the nerve growth factors that normally keep them healthy. Such deprivation might not occur as much in mouse brains as in aged human brains, he says; the team is now studying mice that under-express such growth factors.

Other research groups are studying the N-APP pathway for links to Alzheimer's. David Holtzman, a neuroscientist at Washington University in St Louis, Missouri, is testing blood and spinal fluid from patients with Alzheimer's to see if "this fragment of APP is even present in human body fluids". If it is, Holtzman hopes to find out whether it can be used in diagnosing Alzheimer's or tracking its progress.

Wilcock says that her research group recently developed transgenic mice that have Alzheimer's-like amyloid deposits, neurofibrillary tangles and neuronal loss, and she hopes to study whether the N-APP pathway is driving some of that destruction. "I think it opens up a lot of lines of new research for us," she says. "These are important questions that everybody should be looking at."

Jim Schnabel 